\title{
COVID-19-Compliant Strategies for Supporting Treatment Adherence Among People Living with HIV in Sub-Saharan Africa
}

\author{
Thabani Nyoni ${ }^{1} \cdot$ Moses Okumu ${ }^{2}$
}

Published online: 24 April 2020

(c) Springer Science+Business Media, LLC, part of Springer Nature 2020

COVID-19 public health responses such as social distancing and shelter-in-place protocols are critical to preventing and containing the spread of coronavirus [1]. Yet these important measures may also prevent people living with HIV (PLWH) from accessing needed antiretroviral (ART) treatments and reduce their likelihood of treatment adherence [2-4]. PLWHs from sub-Saharan Africa (SSA) account for about $67.5 \%$ of the 37.9 million PLWHs globally [5]. As care providers and policymakers draw on resources and extant strategies to curb the COVID-19 pandemic, WHO and UNAIDS are calling on governments to develop and implement strategies that minimize disruptions in access and adherence to treatment regimens for PLWHs [6]. Although SSA remains the global region least affected by the COVID19 pandemic, we focus on this region due to its disproportionately large PLWH population and because prior HIV research suggests that the burden of pandemics tends to shift over time to poorer populations and countries due to their comparatively weak health care systems and other related issues [7]. We first outline four ways in which COVID-19 public health responses are stressful to PLWHs and disrupt their access and adherence to ART. Then, drawing on lessons from successful HIV treatment adherence interventions, we detail several ways to harmonize these seemingly dissonant health care efforts in SSA.

All authors contributed equally.

Moses Okumu

mokumu@email.unc.edu

Thabani Nyoni

tnyoni@wustl.edu

1 Brown School of Social Work, Washington University in Saint Louis, 1 Brookings Dr, Saint-Louis, MO 63130, USA

2 School of Social Work, University of North Carolina-Chapel Hill, 325 Pittsboro St., CB\#3550, Chapel Hill, NC 27599, USA

\section{Negative Impacts of COVID-19 Measures on HIV Treatment and ART Adherence in SSA}

\section{Impeding Access to Clinical Care}

To date, 18 African countries-including many with high HIV prevalence (e.g., Lesotho, Malawi, Namibia, South Africa, Uganda, and Zimbabwe) —have implemented lockdown measures [5, 8]. Depending on the country, lockdown measures may include mandatory geographic quarantines, shelter-in-place requirements (except to buy food and access vital health services), closing certain types of businesses, or banning events and gatherings [9]. These social controls can hinder or delay PLWH's access to HIV care and support at hospital or clinic sites. For instance, a study conducted in China found that $32.6 \%$ of PLWHs were at risk of discontinuing ART and about $48.6 \%$ did not know where to get needed medications due to COVID-19 lockdown measures [4]. In SSA, the effect of travel restrictions on PLWHs could be even more detrimental given the long distances they must often travel to access treatment and care due to the limited availability of specialized HIV services, preferences for higher-tiered facilities, and efforts to mitigate social stigma surrounding treatment $[10,11]$.

\section{Impeding Access to Community-Based Support}

Travel restrictions and social distancing may also disrupt the existing community-based ART delivery and psychosocial support strategies that mitigate the negative impacts of barriers to ART adherence (e.g., psychological distress, community stigma, and distance from health care centers) and provide effective ART uptake reminders to PLWH [12]. By impeding PLWH's ability access to ART medication at community venues or by preventing community health workers from making home visits to conduct health and social needs assessments and deliver ART medication [13-17], COVID-19 prevention efforts may come at the cost of PLWH's physical and psychosocial health. 


\section{Overburdening Public Health Providers}

COVID-19 control efforts may also further stretch the already overburdened health systems serving PLWHs in SSA. In SSA, most PLWHs receive treatment and care from poorly resourced public health facilities with inadequate infrastructure and staff shortages [17, 18]. Even in well-resourced treatment contexts (e.g., China), evidence suggests that many PLWHs cannot access their ART medication or face delays in treatment because hospitals are busy treating patients with COVID-19 [4]. Given that most public health facilities in SSA struggled to provide HIV treatment and care before the COVID-19 pandemic, significant numbers of PLWHs will likely face service disruptions unless timely, intentional steps are taken to prevent them.

\section{Increasing Food Insecurity}

Lockdown and social distancing measures will likely disrupt economic activity and thus increase food insecurity - a significant barrier to ART adherence [19, 20]. For instance, in Liberia during the Ebola crisis, rice prices increased by more than $30 \%$ and cassava prices rose to $150 \%$; in China, food prices have increased by $20 \%$ during the COVID-19 pandemic [21]. In Tanzania, PLWHs who rely on periodic farming and cultivation jobs are breaking social distancing requirements to earn money for food, putting this immunocompromised population at even greater risk of COVID-19 infection [19]. To reduce food insecurity, some SSA countries (e.g., Rwanda, Uganda and Nigeria) have combined restrictive measures with food distribution services for vulnerable communities. However, some of these distribution services may not adhere to social distancing guidelines. In fact, in many SSA countries, vulnerable communities congregate at food distribution centers, increasing their risk of acquiring and spreading COVID-19.

COVID-19 prevention and containment strategies, while necessary, are exacerbating difficulties related to the treatment and support of PLWHs in SSA: a vulnerable population in a largely under-resourced environment. We thus identify and discuss three cost-effective strategies for supporting psychosocial health and ART adherence among this population while complying with the current social controls implemented to slow the spread of COVID-19.

\section{COVID-19-Compliant Strategies for Promoting ART Adherence}

\section{Treatment Supporters}

Treatment supporters offer one cost-effective and COVID19-compliant means of providing community-based ART adherence support and care for PLWHs. Nominated by patients or health care providers, treatment supporters are typically PLWH's confidantes (e.g., close relatives, parents, or partners) who have the moral authority to help them with ART management and provide psychosocial support [14-16]. A recent systematic review found that treatment supporter interventions in SSA significantly improved ART adherence and virologic suppression among adult PLWHs compared to the standard of care [16]. Notably, this study also found that treatment supporters selected by PLWHs themselves had comparable effects on ART adherence outcomes to those selected by health care providers.

For several reasons, treatment supporters offer PLWHs an effective and COVID-19-compliant resource. As members of PLWH's home communities, treatment supporters can provide home-based psychosocial support without violating COVID-19 lockdown measures. Their close relationships and at times cohabitation with PLWHs make them the most available, reliable, and convenient source of psychosocial support at a time when options for social contact remain limited. Additionally, treatment supporters are well positioned to support empowered living (i.e., having the necessary motivation, support, and knowledge to maintain optimal ART adherence, even in stressful situations [15]) among PLWHs by mitigating stress that may result from COVID-19 social control measures. To address PLWH's possible forgetfulness, treatment fatigue, and effects of psychological distress, treatment supporters may also assist by collecting and delivering medication, administering medication, and providing clinic attendance reminders $[13,15]$.

For treatment supporters to provide effective psychosocial support that complies with current COVID-19 prevention and containment protocols, capacity strengthening may be required. As suggested by UNAIDS, we posit that public health policies designed to empower and strengthen communities' capacities to support and protect community members' health may have greater individual and communal health benefits than efforts to criminalize breaches of COVID-19 public health policies [22].

\section{Digital Health Interventions}

The exponential growth in the number of mobile phone users in SSA offers a digital venue for individuals and public health organizations to provide psychosocial support and ART adherence messages while complying with COVID-19 social control measures. In 2017, over three-quarters of SSA's population had a mobile phone subscription [23]. Studies conducted in Kenya and Cameroon reported that two-way text messaging supports for PLWH yielded significant improvements in ART adherence and rates of viral suppression [24, 25]. Two-way text messaging allows people in different locations to easily 
check on each other, converse, and provide psychosocial support without requiring travel or physical gathering. Mobile phone texts and calls could also enable providernominated treatment supporters to conduct health assessments while observing social distancing and stay-at-home measures and reducing PLWH's potential exposure to HIV-related community stigma.

\section{Cash Transfers}

During the COVID-19 pandemic, cash transfers could in fact prove more effective than directly distributing food in alleviating food insecurity, increasing options for good nutrition, and increasing financial autonomy among PLWHs. Unlike food distribution services, which often occur at centralized locations requiring people to congregate, cash transfers provide individuals with the means to purchase food while adhering to social distancing requirements. Mobile money platforms, which allow recipients to transfer money electronically without leaving their quarantine space, offers another useful application of digital technology (e.g. mobile phones) under quarantine conditions. Encouragingly, prior HIV treatment literature in SSA suggests that cash transfers are associated with PLWH's ART adherence even when no social distancing regulations exist [26, 27].

As COVID-19 continues to reshape social environments and policy landscapes, countries in SSA can invest in community-based ART treatment supporter services and digital interventions to increase PLWH's access to medication, support, and nutrition. We believe these services would be most effective when deployed in concert. For instance, patient-nominated treatment supporters can assist with medication pickup, delivery, and reminders, while health worker-nominated treatment supporters send text messages about health check-ups and tips for self-care via mobile phones. Non-profit and government organizations supporting PLWHs can leverage mobile money platforms to transfer cash that patient-nominated treatment supporters can use to buy nutritious food for PLWHs. Collectively, these strategies offer a cost-effective and COVID-19-compliant program for promoting the health and ART adherence of PLWHs in SSA even after the present coronavirus pandemic has passed.

\section{References}

1. Lancet T. COVID-19: learning from experience. Lancet. 2020;395(10229):1011. https://doi.org/10.1016/s0140 -6736(20)30686-3.

2. Druss BG. Addressing the COVID-19 pandemic in populations with serious mental illness. JAMA Psychiatry. 2020. https://doi. org/10.1001/jamapsychiatry.2020.0894.
3. Logie CH, Turan JM. How do we balance tensions between COVID-19 public health responses and stigma mitigation? Learning from HIV research. AIDS Behav. 2020. https://doi. org/10.1007/s10461-020-02856-8.

4. Jiang $\mathrm{H}$, Zhou $\mathrm{Y}$, Tang W. Maintaining HIV care during the COVID-19 pandemic. Lancet HIV. 2020. https://doi. org/10.1016/s2352-3018(20)30105-3.

5. UNAIDS. Fact Sheet: World AIDS Day 2019-Global HIV Statistics. UNAIDS. 2019. https://www.unaids.org/sites/defau 1t/files/media_asset/UNAIDS_FactSheet_en.pdf. Accessed 13 Apr 2020.

6. WHO. Mental health and psychosocial considerations during COVID-19 outbreak: tips from World Health Organization. UNICEF. 2020. https://www.unicef.org/bulgaria/en/stories/ mental-health-and-psychosocial-considerations-during-covid -19-outbreak. Accessed 14 Apr 2020.

7. Hargreaves J, Davey C, et al. Three lessons for the COVID-19 response from pandemic HIV. Lancet HIV. 2020. https://doi. org/10.1016/s2352-3018(20)30110-7.

8. Witt A. PRIF Blog. An island of internationalism: the African Union's fight against corona. 2020. https://blog.prif. org/2020/04/07/an-island-of-internationalism-the-african-union s-fight-against-corona/. Accessed 14 Apr 2020.

9. Kaplan J, Frias L, McFall-Johnsen M. A third of the global population is on coronavirus lockdown-here's our constantly updated list of countries and restrictions. Business Insider. 2020. https://www.businessinsider.com/countries-on-lockd own-coronavirus-italy-2020-3. Accessed 14 Apr 2020.

10. Akullian AN, Mukose A, Levine GA, Babigumira JB. People living with HIV travel farther to access healthcare: a population-based geographic analysis from rural Uganda. J Int AIDS Soc. 2016;19(1):20171. https://doi.org/10.7448/ias.19.1.20171.

11. Posse M, Meheus F, Asten HV, Ven AVD, Baltussen R. Barriers to access to antiretroviral treatment in developing countries: a review. Trop Med Int Health. 2008;13(7):904-13. https://doi. org/10.1111/j.1365-3156.2008.02091.x.

12. Nachega JB, Adetokunboh O, Uthman OA, et al. Communitybased interventions to improve and sustain antiretroviral therapy adherence, retention in HIV care and clinical outcomes in lowand middle-income countries for achieving the UNAIDS 90-9090 targets. Curr HIV/AIDS Rep. 2016;13(5):241-55. https://doi. org/10.1007/s11904-016-0325-9.

13. Kunutsor S, Walley J, Katabira E, et al. Improving clinic attendance and adherence to antiretroviral therapy through a treatment supporter intervention in Uganda: a randomized controlled trial. AIDS Behav. 2011;15(8):1795-802. https://doi.org/10.1007/ s10461-011-9927-9.

14. Nachega JB, Knowlton AR, Deluca A, et al. Treatment supporter to improve adherence to antiretroviral therapy in HIVinfected South African adults. J Acquir Immune Defic Syndr. 2006;43(Supp1):S127-S133133. https://doi.org/10.1097/01. qai.0000248349.25630.3d.

15. Nakamanya S, Muyanga BN, Muhumuza R, Bukenya D, Seeley J. Are treatment supporters relevant in antiretroviral long-term therapy (ART) adherence? Experiences from a long-term ART cohort in Uganda. Glob Public Health. 2019;14(3):469-80. https://doi.org/10.1080/17441692.2018.1514418.

16. Nyoni T, Sallah YH, Okumu M, Byansi W, Lipsey K, Small E. The effectiveness of treatment supporter interventions in antiretroviral treatment adherence in sub-Saharan Africa: a systematic review and meta-analysis. AIDS Care. 2020. https://doi. org/10.1080/09540121.2020.1742870.

17. Dahab M, Charalambous S, Karstaedt AS, et al. Contrasting predictors of poor antiretroviral therapy outcomes in two South African HIV programmes: a cohort study. BMC Public Health. 2010;10(1):430. https://doi.org/10.1186/1471-2458-10-430. 
18. Vyankandondera J, Mitchell K, Asiimwe-Kateera B, et al. Antiretroviral therapy drug adherence in Rwanda: perspectives from patients and healthcare workers using a mixed-methods approach. AIDS Care. 2013;25(12):1504-12. https://doi.org/10.1080/09540 121.2013.779626.

19. Ashly J. 'We feel abandoned': HIV positive Tanzanians brace for COVID-19. ALJAZEERA. 2020. https://www.aljazeera.com/ news/2020/04/feel-abandoned-hiv-positive-tanzanians-brace -covid-19-200413132301763.html. Accessed 14 Apr 2020.

20. Singer AW, Weiser SD, Mccoy SI. Does food insecurity undermine adherence to antiretroviral therapy? A systematic review. AIDS Behav. 2014;19(8):1510-26. https://doi.org/10.1007/s1046 1-014-0873-1.

21. Choularton R, Mallory M. How to address the impact of COVID19 on global food systems. Devex. 2020. https://www.devex.com/ news/sponsored/opinion-how-to-address-the-impact-of-covid -19-on-global-food-systems-96892. Accessed 14 Apr 2020.

22. UNAIDS. Rights in the Time of COVID-19-Lessons from HIV for an Effective, Community-Led Response. UNAIDS. 2020. https ://www.unaids.org/sites/default/files/media_asset/human-right s-and-covid-19_en.pdf. Accessed 13 Apr 2020.

23. Logan S. Study provides fresh insights into the benefits of mobile money in Kenya. The Conversation. 2017. https://theconversation .com/study-provides-fresh-insights-into-the-benefits-of-mobil e-money-in-kenya-70432. Accessed 14 Apr 2020.

24. Bigna JJR, Noubiap JJN, Kouanfack C, Plottel CS, Koulla-Shiro $\mathrm{S}$. Effect of mobile phone reminders on follow-up medical care of children exposed to or infected with HIV in Cameroon (MORE CARE): a multicenter, single-blind, factorial, randomized controlled trial. Lancet Infect Dis. 2014;14(7):600-8. https://doi. org/10.1016/s1473-3099(14)70741-8.

25. Lester RT, Ritvo P, Mills EJ, et al. Effects of a mobile phone short message service on antiretroviral treatment adherence in Kenya (WelTel Kenya1): a randomized trial. Lancet. 2010;376(9755):1838-45. https://doi.org/10.1016/s0140 -6736(10)61997-6.

26. Cluver LD, Toska E, Orkin FM, et al. Achieving equity in HIVtreatment outcomes: can social protection improve adolescent ART-adherence in South Africa? AIDS Care. 2016;28(sup2):7382. https://doi.org/10.1080/09540121.2016.1179008.

27. Czaicki NL, Mnyippembe A, Blodgett M, Njau P, Mccoy SI. It helps me live, sends my children to school, and feeds me: a qualitative study of how food and cash incentives may improve adherence to treatment and care among adults living with HIV in Tanzania. AIDS Care. 2017;29(7):876-84. https://doi. org/10.1080/09540121.2017.1287340.

Publisher's Note Springer Nature remains neutral with regard to jurisdictional claims in published maps and institutional affiliations. 